\title{
Premature coronary artery disease and testosterone in Sri Lankan men
}

\author{
C M Wickramatilake ${ }^{1}$, M R Mohideen ${ }^{2}$, C Pathirana ${ }^{1}$ \\ Sri Lanka Journal of Diabetes, Endocrinology and Metabolism 2014; 4: 17-21
}

\begin{abstract}
Objective: Recent studies have shown that low endogenous testosterone in men is associated with coronary artery disease (CAD) and its risk factors such as obesity, hypertension, dyslipidaemia, and diabetes mellitus. This study was planned to investigate the association of low total testosterone levels with premature coronary artery disease.
\end{abstract}

Methods: Thirty one men, 45 years of age or below with documented coronary artery disease (mean age $41 \pm 3$ ) constituted the cases. Control group consisted of 31 men below the same age, without having clinically evident coronary artery disease (mean age $37 \pm 4$ ). Total testosterone, fasting plasma glucose and serum lipid levels were measured.

Results: Mean ages of the two groups were significantly different $(p=0.001)$. Body mass index $(p=$ $0.843)$ and hip circumferences $(p=0.097)$ were not significantly different between the two groups, but waist circumference $(p=0.007)$ and waist to hip ratio $(p=0.002)$ were significantly higher among cases. Prevalence of hypertension, diabetes mellitus, and smoking among cases was higher compared to controls. Total testosterone levels of cases were significantly lower than those of controls $(11.1 \pm 3.2$ $\mathrm{nmol} / \mathrm{L}$ vs. $27.1 \pm 4.3 \mathrm{nmol} / \mathrm{L}, \mathrm{p}=0.001)$, which remained significant, following adjustment for the clinical covariates (age, BMI, smoking, diabetes mellitus). Plasma glucose $(p=0.016)$ and HDL-cholesterol $(p=0.001)$ were significantly different between the groups.

Conclusion: Serum total testosterone was significantly lower in patients with premature CAD compared to controls. Low level of total testosterone may be related to the development of premature coronary artery disease.

Keywords: premature coronary artery disease; testosterone; men

\section{Introduction}

The age-standardized death rates for coronary heart disease are declining in many developed countries, but are increasing in developing countries such as in Sri Lanka with demographic changes, urbanization, and lifestyle changes $(1,2)$. Coronary artery disease (CAD) is one of the leading causes of mortality in men. Cardiovascular disease accounts for the highest rate of hospital deaths in Sri Lanka (2). Men of similar age tend to have higher incidence of CAD than premenopausal women (3). Hence, it is fair to assume that androgens may have a role in the development of CAD. In contrast, epidemiological studies have identified an inverse association between testosterone and coronary artery disease in men $(4,5,6)$ with reduced testosterone concentrations being linked to premature CAD (7). There are studies indicating that a low testosterone level is related to the risk factors of cardiovascular disease as well $(8,9,10)$.
Nevertheless, the relationship between testosterone and CAD has not been established yet. Moreover, the relationship between premature CAD and testosterone levels is not well studied. There are no previous studies of this nature conducted in Sri Lanka. Therefore this study was done to investigate whether low total testosterone levels are associated with premature CAD.

\section{Materials and methods}

The research project was approved by the Ethical Review Committee of Faculty of Medicine, University of Ruhuna, Galle, Sri Lanka. Thirty one men of 45 years of age or below with documented coronary artery disease constituted the cases. It included 11 with angiogram proven coronary artery disease patients who were awaiting coronary artery bypass graft at Cardiothoracic Unit, Teaching Hospital, Karapitiya and 20 with confirmed ST- 
elevated myocardial infarction patients admitted to Coronary Care Unit, Teaching Hospital, Karapitiya. The control group consisted of 31 men below the same age, without having clinically evident coronary artery disease. They were selected from the patients who were awaiting minor surgery at surgical units, Teaching Hospital, Karapitiya.

Data were collected using an intervieweradministered questionnaire, after the informed written consent. Anthropometric measurements were obtained according to the standard protocols. Fasting morning blood samples were collected for biochemical assays. Serum testosterone levels were estimated using commercial enzyme immuno assay kit (Pathozyme Testosterone, Omega Diagnostics Ltd, Omega House, UK). Concentrations of fasting plasma glucose, serum total cholesterol, high-density lipoprotein cholesterol and triglyceride levels were measured by colourimetric methods using commercial kits (ProDia International, UAE).

Data were analyzed using Minitab version 15 for Windows. Categorical data were displayed as percentages and frequencies. Categorical data were analyzed using the Chi-squared test or Fisher's exact test. Numerical data were examined for normality and presented as mean \pm SD. Adjusted means of hormone, lipid levels were calculated using multiple regressions. Age, body mass index, smoking and diabetes were used as independent predictor variables for testosterone and age, BMI, smoking, diabetes and use of statin were used as independent variables for lipids and for plasma glucose; age and BMI were used. Comparison between cases and controls was analyzed using two sample t-tests for independent samples assuming unequal variance. Pearson correlation coefficient was used to assess the relationship between serum testosterone concentrations and other risk factors.

\section{Results}

The age of the CAD group ranged from 32 to 45 years and the age of control group varied from 30 to 45 years. Mean ages of the two groups were significantly different $(41 \pm 3$ vs. $37 \pm 4$ years, $p=0.001)$. Although BMI $(23.6 \pm 4$ vs. $23.5 \pm 6, p=0.906)$ and hip circumferences ( $89.7 \pm 8$ vs. $86.3 \pm 8 \mathrm{~cm}, \mathrm{p}=0.133$ ) were not significantly different between the two groups, waist circumference (84.3 \pm 10 vs. $76.7 \pm 11 \mathrm{~cm}, \mathrm{p}=0.01)$ and the waist-hip ratio (0.93 \pm 0.04 vs. $0.88 \pm 0.06, p=0.001$ ) were significantly different (Table 1).

Prevalence of hypertension, diabetes mellitus and history of statin use among the cases were 8 (25.8 \%), 4 (12.9\%), 13 (41.9\%) respectively, while none reported in the control group. Smoking was reported in 23 (74.2\%) cases; 15 of them were current smokers and eight were exsmokers for approximately three months at the time of recruitment to the study. Six (19.3\%) controls were pastsmokers and they had stopped smoking for more than one year by the time of recruitment to the study (Table 1).

Table 1. Baseline characteristics in cases vs. controls

\begin{tabular}{lccc}
\hline Characteristics & Cases $(n=31)$ & Control group $(n=31)$ & $p$ \\
\hline Age (in years) & $41 \pm 3$ & $37 \pm 4$ & 0.001 \\
BMI $\left(\right.$ Kgm $\left.^{-2}\right)$ & $23.6 \pm 4$ & $23.4 \pm 6$ & 0.843 \\
Waist circumference $(\mathrm{cm})$ & $84.3 \pm 10$ & $76.5 \pm 11$ & 0.007 \\
Hip $(\mathrm{cm})$ & $89.7 \pm 8$ & $85.9 \pm 8$ & 0.097 \\
W-H ratio & $0.93 \pm 0.04$ & $0.88 \pm 0.06$ & 0.002 \\
SBP mmHg & $134 \pm 28$ & $123 \pm 13$ & 0.042 \\
DBP mmHg & $83 \pm 18$ & $77 \pm 7$ & 0.090 \\
Diabetes mellitus & $4(12.9 \%)$ & Not present & - \\
Hypertension & $8(25.8 \%)$ & Not present & - \\
Smoking & $23(74.2 \%)$ & $6(18.7 \%)$ & 0.001 \\
Statin treatment & $13(41.9 \%)$ & Not used & - \\
\hline
\end{tabular}

$\mathrm{BMI}=$ Body mass index, $\mathrm{W}-\mathrm{H}$ ratio $=$ Waist to hip ratio, $\mathrm{SBP}=$ Systolic blood pressure, $\mathrm{DBP}=$ Diastolic blood pressure. Data presented as mean $\pm \mathrm{SD}$, percentages or frequencies, p-Value calculated using two-sample t-test. Other p values calculated using Chisquared test or Fisher's exact test. 
Serum total testosterone concentration (TT) among the cases was significantly lower than the controls ( $p=0.001)$ which remained significant $(p=0.001)$ even after adjustments for clinical covariates (age, BMI, smoking, diabetes mellitus).

The concentrations of total cholesterol (T-Ch), LDLcholesterol (LDL-Ch) and triglycerides (TGs) were not significantly different between the two groups, but after the adjustments for the clinical covariates (age, BMI, smoking, diabetes mellitus and use of statin), the difference between the two groups became significant; T-Ch ( $p=0.004)$, LDL-Ch $(p=0.001)$, TGs $(p=0.001)$. Serum HDL-Cholesterol (HDL-Ch) was significantly different between two groups (Table 2). Plasma glucose concentration in the CAD group was significantly higher compared to the controls ( $p=0.016$ ), which became stronger $(p=0.001)$ after the adjusting for age and BMI.

A significant positive correlation was observed between total testosterone and the HDL-Ch ( $\mathrm{p}=0.001)$. A negative correlation existed between total testosterone and age $(\mathrm{p}=0.001)$, waist circumference $(\mathrm{p}=0.008), \mathrm{W}-\mathrm{H}$ ratio $(\mathrm{p}=0.003$ ) showed highly significant (all $\mathrm{p}<0.05$ ), while modest significance was present between TT and hip circumference ( $p=0.087)$, SBP $(p=0.067)$, DBP $(\mathrm{p}=0.057)$ and relationship was not significant with BMI ( $p=0.869)$. The negative correlations between TT and TCh $(p=0.308)$, TGs $(p=0.229)$ and PG $(p=0.115)$ were non significant, while modest significance was present with LDL-Ch $(\mathrm{p}=0.065)$.

Table 2. Comparison of laboratory findings

\begin{tabular}{|c|c|c|c|c|c|c|}
\hline Measurements & $\begin{array}{c}\text { Patients } \\
(n=31) \\
\text { unadjusted }\end{array}$ & $\begin{array}{l}\text { Controls } \\
(n=31) \\
\text { unadjusted }\end{array}$ & $p$ & $\begin{array}{c}\text { Patients } \\
(n=31) \\
\text { adjusted }^{*}\end{array}$ & $\begin{array}{l}\text { Controls } \\
(n=31) \\
\text { adjusted }\end{array}$ & $p$ \\
\hline TT (nmol/L) & $11.1 \pm 3.2$ & $27.1 \pm 4.3$ & 0.001 & $11.1 \pm 1.2$ & $27.0 \pm 3.2$ & 0.001 \\
\hline $\mathrm{T}-\mathrm{Ch}(\mathrm{mmol} / \mathrm{L})$ & $4.7 \pm 2$ & $5.2 \pm 1.3$ & 0.34 & $4.7 \pm 0.7$ & $5.2 \pm 0.3$ & 0.004 \\
\hline HDL-Ch $(\mathrm{mmol} / \mathrm{L})$ & $1.0 \pm 0.4$ & $1.7 \pm 0.7$ & 0.001 & $1.0 \pm 0.2$ & $1.7 \pm 0.3$ & 0.001 \\
\hline LDL-Ch(mmol/L) & $3.7 \pm 1.8$ & $3.1 \pm 0.4$ & 0.13 & $3.6 \pm 0.7$ & $3.1 \pm 0.1$ & 0.001 \\
\hline TGs (mmol/L) & $2.0 \pm 1$ & $1.6 \pm 0.8$ & 0.11 & $2.0 \pm 0.3$ & $1.6 \pm 0.08$ & 0.001 \\
\hline $\mathrm{PG}(\mathrm{mmol} / \mathrm{L})$ & $6.2 \pm 2.5$ & $4.9 \pm 0.6$ & 0.016 & $6.2 \pm 0.6$ & $4.9 \pm 0.2$ & 0.001 \\
\hline
\end{tabular}

$\mathrm{TT}=$ total testosterone, $\mathrm{TG}$ = triglycerides, $\mathrm{TCh}=$ total cholesterol, HDL-Ch $=$ high-density lipoprotein cholesterol, $\mathrm{LDL}-\mathrm{Ch}=$ low density lipoprotein cholesterol, $\mathrm{PG}=$ plasma glucose. All values expressed as mean $\pm \mathrm{SD}$. p-Values stated calculated by two-sample t-test. * The measurements were adjusted for clinical covariate in the regression analysis.

Table 3. Relationships between testosterone levels and other measurements

\begin{tabular}{lccc}
\hline Measurements & TT & Measurements & TT \\
\hline Age & $-0.593(0.001)$ & TGs & $-0.155(0.229)$ \\
BMI & $-0.021(0.869)$ & TCh & $-0.132(0.308)$ \\
Waist & $-0.332(0.008)$ & HDL-Ch & $0.719(0.001)$ \\
Hip & $-0.219(0.087)$ & LDL-Ch & $-0.236(0.065)$ \\
W-H ratio & $-0.374(0.003)$ & PG & $-0.202(0.115)$ \\
SBP & $-0.235(0.067)$ & DBP & $-0.243(0.057)$ \\
\hline
\end{tabular}

Number outside the parentheses represents value of correlation coefficient; $\mathrm{p}$ values for correlation coefficient are shown in the parentheses. Abbreviations as in Table1, 2. 


\section{Discussion}

The current study reveals that serum total testosterone concentration was significantly lower in patients with premature coronary artery disease compared to the controls. This finding is consistent with the previous studies suggesting that low testosterone may be a risk factor for the development of $\operatorname{CAD}(4,5,6)$. Furthermore, a recent study done on Turkish subjects has linked the presence of low testosterone to the development of premature CAD, where both total and free testosterone found to be low in the cases compared to controls (7). Findings of our study are in agreement of the above study.

Prevalence of conventional risk factors such as diabetes mellitus, hypertension, hyperlipidaemia and smoking were more prevalent in the premature $\mathrm{CAD}$ group. Although BMI was not significantly different between the two groups, WC and $\mathrm{W}-\mathrm{H}$ ratio were higher in the CAD group reflecting the relationship of central obesity with the development of $\operatorname{CAD}(8,9,10)$.

HDL-Ch level was significantly higher in the control group compared to cases and also showed a significant positive correlation with testosterone. Total testosterone showed modestly significant negative correlation with LDL-Ch. These findings are in agreement with the previous reports, which had shown positive correlation of testosterone with HDL-Ch in healthy men. One proposed mechanism is that testosterone acts through modulation of lipid profile, supported by findings of a favourable relationship with HDL-Ch $(11,12,13)$.

There are studies where intramuscular administration of near-physiological doses of testosterone resulted in a decrease in total and LDL cholesterol levels without significantly affecting HDL cholesterol levels (14-15). Testosterone supplementations in men with hypogonadotropic hypogonadism were shown to increase levels of total and LDL cholesterol, but increased the HDL /LDL ratio (16).

\section{Conclusion}

The serum total testosterone was significantly lower in patients with premature coronary artery disease compared to controls. There was a significant positive association between serum total testosterone and HDL$\mathrm{Ch}$ in both groups. This reflected that low levels of testosterone appear to be related to the development of atherogenic lipid profile and premature coronary artery disease. It is recommended that larger studies are needed to confirm these findings in future.

Conflicts of interest: The authors declared that they had no conflicts of interest concerning this article.

\section{Acknowledgement}

We wish to acknowledge the University Grants Commission, Sri Lanka for the financial assistance provided for the project, Mrs. DABN Amerasekara, (Statistician, Applied Statistic Association, Sri Lanka), Senior Lecturer, Department of Crop Science, Faculty of Agriculture, University of Ruhuna, Sri Lanka for the statistical advice provided, and Mrs. Kawmadhi Abeywaradana, Technical Officer, Department of Biochemistry, Faculty of Medicine, University of Ruhuna, Sri Lanka for the support offered in the biochemical assays.

\section{References}

1. Rosamond W, Flegal K, Furie K, et al. Heart disease and stroke statistics - 2008 update: a report from the American Heart Association Statistics Committee and Stroke Statistics Subcommittee. Circulation 2008; 117: e25-e146.

2. Annual Health Bulletin, Ministry of Health, Sri Lanka 2007; pp 31.

3. Douglas PS. Coronary artery disease in women. In: Braunwald E, Zipes DP, Libby P, eds. Heart disease: a textbook of cardiovascular medicine. Philadelphia, PA: W B Saunders Co, 1997: 1704.

4. Rosano GMC, Sheiban I, Massaro R, et al. Low testosterone levels are associated with coronary artery disease in male patients with angina. Int J Impot Res 2007; 19: $176-182$.

5. Hu X, Rui L, Zhu T, et al. Low testosterone level in middle-aged male patients with coronary artery disease. Eur J Intern Med 2011; 22: e133 -e136.

6. English KM, Mandour O, Steeds RP, Diver MJ, Jones $\mathrm{TH}$, Channer KS. Men with coronary artery disease have lower levels of androgens than men with normal coronary angiograms. Eur Heart J 2000; 21: 890-4.

7. Turhan S, Tulunay C, Gulec S, et al. The association between androgen levels and premature coronary artery disease in men. Coron Artery Dis 2007; 18: 159-62.

8. Phillips GB, Jing TY, Resnick LM, Barbagallo M, Laragh JH, Sealey JE. Sex hormones and haemostatic risk factors for coronary heart disease in men with hypertension. $J$ Hypertens 1993; 11: 699-702.

9. Zumoff B, Strain GS, Miller LK, et al. Plasma free and non sex hormone binding globulin bound testosterone is decreased in obese men in proportion to their degree of obesity. J Clin Endocrinol Metab 1990; 71: 929-31.

10. Laaksonen DE, Niskanen L, Punnonen K, et al. Testosterone and sex hormone-binding globulin predict the metabolic syndrome and diabetes in middle-aged men. Diabetes Care 2004 ; 27(5): 1036-41.

11. Van Pottelbergh I, Braeckman L, De Bacquer D, De Backer G, Kaufman JM. Differential contribution of testosterone 
and estradiol in the determination of cholesterol and lipoprotein profile in healthy middle-aged men. Atherosclerosis 2003; 166: 95-102.

12. Zmuda JM, Cauley JA, Kriska A, Glynn NW, Gutai JP, Kuller LH. Longitudinal relation between endogenous testosterone and cardiovascular disease risk factors in middle-aged men. A 13-year follow-up of former multiple risk factor intervention trial participants. Am J Epidemiol 1997; 146: 609-17.

13. Khaw KT, Barrett-Connor E. Endogenous sex hormones, high density lipoprotein cholesterol and other lipoprotein fractions in men. Arterioscler Thromb 1991; 11: 489-94.
14. Zgliczynski S, Ossowski M, Slowinska-Srzednicka J, et al. Effect of testosterone replacement therapy on lipids and lipoproteins in hypogonadal and elderly men. Atherosclerosis 1996; 121: 35-43.

15. Morley JE, Perry HM, Kaiser FE, et al. Effects of testosterone replacement in old hypogonadal males: a preliminary study. J Am Geriatr Soc 1993; 41: 149-52.

16. Ozata M, Yildirimkaya M, Bulur M, et al. Effects of gonadotropin and testosterone treatments on lipoprotein (a), high density lipoprotein particles, and other lipoprotein levels in male hypogonadism. J Clin Endocrinol Metab 1996; 81: 3372-8. 